\title{
Uso de elementos de protección personal en Unidad de Emergencia HBLT en contexto pandemia COVID-19
}

\author{
Use of personal protection elements in the HBLT Emergency Unit in the \\ context of the COVID-19 pandemic
}

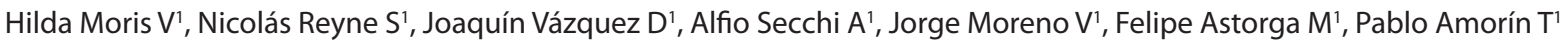

\section{Resumen}

Los elementos de protección personal (EPP) son el conjunto de equipamiento, componente de las precauciones estándares, destinados a proteger la piel y mucosas del operador evitando el contacto con agentes infecciosos. Los funcionarios de salud que tratan con pacientes COVID-19 se encuentran más expuestos a infectarse en comparación con el resto de los funcionarios de salud, razón por la cual deben utilizar de manera adecuada estos elementos de protección, para protegerse de una posible infección por COVID-19. El objetivo del estudio es determinar disponibilidad, manejo y uso EPP utilizados por los funcionarios del Servicio de Urgencia del Complejo Asistencial Barros Luco Trudeau. Este es un estudio transversal descriptivo realizado por medio de encuesta a los funcionarios de la Unidad de Emergencia HBLT a través de Google Forms ${ }^{\circledast}$. Del total de funcionarios de la Unidad de Emergencia del Hospital Barros Luco Trudeau, un total de 123 funcionarios realizaron la encuesta cumpliendo con los criterios de inclusión, de los cuales 78 fueron mujeres y 45 hombres, con edades dentro del rango desde los 20 años a mayores de 61 años. Se observó que 39,0\% de los funcionarios fue notificado como COVID-19 positivo. Un 60,2\% de los funcionarios tuvo una secuencia predeterminada para instalación de los EPP y un 68,3\% para el retiro de los EPP. Los más utilizados fueron mascarillas quirúrgicas (95,9\%), mascarilla KN 95 (88,6\%) y guantes (90,2\%). Este estudio contribuye a destacar la importancia en la correcta utilización de los EPP por parte de los funcionarios de la salud, para limitar los contagios y mejorar el cuidado de los equipos de salud durante la pandemia de COVID-19. Además, se describir un patrón de sintomatología más frecuente en los profesionales sanitarios del servicio de urgencia.

Palabras clave: COVID-19; SARS-CoV-2; elementos de protección personal; trabajadores de la salud.

\begin{abstract}
Personal protective equipment (PPE) is a set of equipment, a component of the standard precautions, intended to protect the skin and mucous membranes of the operator avoiding contact with infectious agents. Healthcare workers who deal with COVID-19 patients are at higher risk of being infected compared to other health professionals. Thus they must use them appropriately to protect themselves from possible COVID-19 infection. This study aimed to determine the presence, handling, and use of PPE by the personnel of the Emergency Service of the Barros Luco Trudeau Department. This descriptive cross-sectional study was carried out applying a survey to the staff of the HBLT Emergency Unit through the Google Forms ${ }^{\circledR}$ platform. A total of 123 staff of the Emergency Unit of the Barros Luco Trudeau Hospital that answered the survey met the inclusion criteria. Seventy-eight of them were women and 45 men, within the range of 20 to over 61 years old. Thirty-nine per cent of the personnel were notified as COVID-19 positive. Regarding PPE use, 60,2\% of the personnel had a predetermined sequence for installation, and $68.3 \%$ had a correct sequence for removal of the PPE. The most used were surgical masks (95.9\%), KN 95 masks (88.6\%) and gloves (90.2\%). Of those COVID-19 positive $46.3 \%$ were asymptomatic, and of those who had symptoms the most prevalent ones were headache (41.5\%), tiredness (34.1\%) and odynophagia (31.7\%). This study highlights the importance of using the personal protective elements by health staff as self-care during the COVID-19 pandemic, in addition to describing the most frequent pattern of symptoms in health professionals in the emergency department.
\end{abstract}

Keywords: COVID-19: SARS-CoV-2; personal protective equipment; health personnel.

Fecha de envío: 2021-06-16 - Fecha de aceptación: 2021-09-29

(1) Unidad de Emergencia Hospital Barros Luco Trudeau. Santiago, Chile.

Autor de correspondencia franmorisv@gmail.com 


\section{Introducción}

A finales de diciembre de 2019, un grupo de pacientes ingresaron a diversos hospitales con un diagnóstico inicial de neumonía de etiología desconocida, en Wuhan, provincia de Hubei, China (Bogoch et al., 2020; Lu et al., 2020; Rothan et al., 2020). Después de algunas semanas fue identificado como agente patógeno al SARS-CoV-2, un agente patógeno que ingresa principalmente por medio del tracto respiratorio, que produce la enfermedad conocida como COVID-19.

Los brotes descritos en la literatura de otras variantes de coronavirus (CoV) incluyen el síndrome respiratorio agudo severo SARS-CoVy el síndrome respiratorio del medio oriente MERS-CoV, que se han caracterizado como agentes de gran amenaza para la salud pública (Li et al., 2020; Rothan et al., 2020).

Los síntomas de la infección por SARS-CoV-2 aparecen después de un período de incubación de aproximadamente 5 días (Li et al., 2020; Rothan et al., 2020), pudiendo cursar con recuperación de los síntomas en promedio a los 14 días o complicándose, llegando hasta la muerte en un periodo entre 6 a 41 días, con una mediana de 14 días (Rothan et al., 2020; Wang et al., 2020). La extensión de este período depende principalmente de la edad del paciente y del estado del sistema inmunitario de éste, siendo más corto en pacientes mayores de 70 años en comparación con los menores de 70 años (Rothan et al., 2020; Wang et al., 2020).

Los síntomas más frecuentes al inicio de la enfermedad son fiebre sobre $37,8^{\circ} \mathrm{C}$, tos seca y fatiga, mientras que otros síntomas incluyen producción de esputo, dolor de cabeza, hemoptisis, diarrea, disnea y linfopenia. Otros signos que se ha ido encontrando son: disgeusia, anosmia, vómito y mialgia generalizada. Características clínicas reveladas en imagenología por medio de tomografía computarizada de tórax son coincidentes con los de neumonía (Carlos et al., 2020; Huang et al., 2020; Ren et al., 2020; Rothan et al., 2020; Wang et al., 2020).

Esfuerzos e indicaciones han sido propuestos e implementados con el fin de proteger de contagio a poblaciones susceptibles, tales como personas mayores, proveedores de atención médica y niños (Cook, 2020).

Los elementos de protección personal (EPP) son el conjunto de elementos de equipamiento, componente de las precauciones estándares, destinados a proteger la piel y mucosas del operador en forma de barreras y evitar que se ponga en contacto con los agentes infecciosos o fluidos, fómites, superficies u otros componentes ambientales donde pueden encontrarse los agentes (Luzi \& Radaelli, 2020).
Los trabajadores sanitarios que tratan a pacientes con infecciones COVID-19 se encuentran más expuestos a infectarse, razón cual deben utilizar EPP para protegerse de las gotitas de Flügge u otros fluidos corporales de los pacientes infectados y de las superficies contaminadas (Jin et al., 2020)

Dentro de los EPP se incluye delantales, batas o monos (de una sola pieza), guantes, mascarillas y equipamiento de respiración (respiradores) y gafas protectoras. Estos elementos se deben poner de forma correcta; y existe la posibilidad que los usuarios se contaminen al quitárselos (Jin et al., 2020). Sólo el uso apropiado de EPP reduce significativamente el riesgo de transmisión viral (Hellewell, et al., 2020). La Unidad de Emergencia (SU) del Complejo Asistencial Barros Luco Trudeau, ubicada en la comuna de San Miguel, centro urbano de alta complejidad, base del Servicio de Salud Metropolitano Sur (SSMS) de la ciudad de Santiago, Chile, alberga la atención de aproximadamente 1 millón 158 mil personas. En este complejo asistencial trabajan un total de 4000 personas aproximadamente, de los cuales en el servicio de urgencia prestan función 336 personas con diversas profesiones y tareas entre los que se encuentran médicos(as), odontólogos(as), enfermeras(os), kinesiólogos (as) técnicos (as) en enfermería (TENS) y en técnicos (as) en odontología (TONS).

Una de las causas de contagio del personal de salud y diseminación del virus es la grave falta de información y el incorrecto uso de EPP durante la atención. Los esfuerzos actuales para la contención de la pandemia no son totalmente efectivos. Sin embargo, podemos esperar que estos esfuerzos retrasen la propagación de SARS CoV2 y nos brinden el tiempo suficiente para desarrollar vacunas y agentes antivirales eficaces contra el virus (Verbeek et al., 2020).

El objetivo principal de este estudio fue determinar la disponibilidad, manejo y uso de EPP utilizados por los funcionarios del servicio de urgencia del Complejo Asistencial Barros Luco Trudeaud desde el inicio de la pandemia COVID-19. Como objetivo secundario se evaluó los síntomas más frecuentes entre el personal contagiado en este servicio.

\section{Materiales y métodos}

Se realizó un estudio transversal descriptivo cuantitativo donde se evaluaron los objetivos de este estudio a través de una encuesta aplicada a los funcionarios de la SU.

Se incluyeron a los funcionarios que se desempeñaron en la SU durante el periodo de Emergencia Sanitaria producto de pandemia por SARS-CoV2, durante los meses de agosto de 2020 a febrero 2021. 
La encuesta fue realizada por los investigadores y fue aplicada a los funcionarios por medio de la plataforma Google Forms ${ }^{\circledR}$, previa firma de consentimiento informado para participar del trabajo.

Las variables evaluadas fueron: género, nacionalidad, edad, profesión, patologías de base, hábitos, contacto con pacientes COVID positivo, sintomatología asociada a posible contagio COVID, uso de test rápido, uso de test $\mathrm{PCR}$, confirmación de caso COVID positivo, uso de EPP, origen de EPP, secuencia de instalación y retiro de los EPP y percepción acerca del Centro de Salud.

I. Criterios de selección de la muestra

A. Criterios de Inclusión:

- Funcionarios clínicos y administrativos de la Unidad de Emergencia del Complejo Asistencial Barros Luco.

- Funcionarios con atención directa a pacientes COVID-19 en el Servicio de Emergencias.

B. Criterios de Exclusión:

- Funcionarios cuyo oficio no fue prestado a la Unidad de Emergencia del Complejo Asistencial Barros Luco.

- Funcionarios cuya encuesta se encuentre incompleta.

II. Instrumentos de recolección de datos

Los datos fueron extraídos de la encuesta, digitados y analizados en una planilla Microsoft Excel ${ }^{\mathrm{TM}}$. Se utilizaron estimadores puntuales de porcentaje (\%), media (md) y desviación estándar (ds) para representar las variables.

\section{Análisis estadístico}

Los resultados fueron analizados mediante chi-cuadrado (considerando heterogeneidad de variables), para la asociación de variables nominales, y se consideró que existían diferencias significativas si $P$ $<0,05$. Los programas utilizados para el análisis estadístico fueron Microsoft Excel y Stata v 16.1 (StataCorp, 2019).

\section{Aspectos éticos}

Este estudio fue evaluado y aprobado por Comité Ético Científico del Servicio de Salud Metropolitano Sur. En este estudio no se incluyeron datos de identificación personal de los funcionarios. La participación de los funcionarios que prestan servicios a la Unidad de Emergencia del Complejo Asistencial Barros Luco en este estudio fue completamente voluntaria. Previo a la aplicación de la encuesta los participantes firmaron un consentimiento informado donde autorizaban el uso de la información en trabajos de investigación.

\section{Resultados}

Del total de funcionarios de la Unidad de Emergencia del Hospital Barros Luco Trudeau (336 personas), un total de 123 individuos completaron la encuesta y cumplieron con los criterios de inclusión, de los cuales 78 fueron mujeres y 45 hombres (Tabla 1), con edades entre 20 y 61 años.

En relación con la distribución de la profesión u ocupación actual de los diferentes funcionarios se observó que la mayor participación en completar la encuesta fue por parte de TENS/TONS $(29,3 \%)$ y enfermeros (as) (20,3\%).

En la tabla 2 se muestra la distribución de patologías de base y hábitos entre los funcionarios del servicio. Se observa que la mayoría de los funcionarios no presentaban patologías de base. Cerca de un $26,0 \%$ de los funcionarios presentan patologías crónicas como diabetes, hipertensión, asma, entre otras. Llama la atención que un $56,9 \%$ eran fumadores.

Al analizar el contacto de los funcionarios con individuos COVID-19 positivo dentro de la Unidad de Emergencia, se observa que el 91,1\% de los funcionarios (112 funcionarios) tuvieron relación con individuos positivos a la infección (Tabla 3).

Por otro lado, 39,0\% de ellos fue notificado como COVID-19 positivo. Al analizar los datos en relación con los signos y síntomas presentados en funcionarios sospechosos de COVID-19, se observó que $46,3 \%$ no presentó ningún síntoma. No obstante, al considerar sintomatología prevalente según lo establecido por la OMS fueron dolor de cabeza (41,5\%), seguido de cansancio (34,1\%), odinofagia $(31,7 \%)$ y en menor proporción anosmia (0,8\%). (Tabla 4).

Hubo gran variabilidad en la utilización de test por Inmunoglobulinas y PCR. El test rápido fue realizado en $79,7 \%$ de los funcionarios, en su mayoría solo una vez (43,9\%). No obstante, el test de PCR fue realizado en $72,4 \%$ de la población objetivo, con frecuencia de ejecución en la mayoría de solo una vez $(37,4 \%)$ (Tabla 5).

En relación con el uso de los EPP, más de la mitad de los funcionarios tuvo una secuencia predeterminada para instalación $(60,2 \%)$ y retiro $(68,3 \%)$ de los EPP. Entre los elementos más utilizados están la mascarilla quirúrgica (95,9\%), mascarilla KN 95 (88,6\%) y guantes $(90,2 \%)$. Además, el $58,5 \%$ de la población en estudio indicó que los EPP fueron proveídos por la institución a la cual prestan servicios (Tabla 6). El 85,4\% de los funcionarios califica a la Institución en relación con los EPP proveídos para protegerlos de la pandemia COVID-19 con nota superior y/o igual a 5 y un 14,6\% con nota 4 y/o inferior a ésta. 
Moris et al.

Tabla 1: Distribución de funcionarios por edad, sexo, profesión y nacionalidad Unidad de Emergencia, Hospital Barros Luco Trudeau. 2020. $n=123$

\begin{tabular}{|c|c|c|c|c|c|}
\hline Edad (años) & $\mathbf{n}$ & $\%$ & Sexo & $\mathbf{n}$ & $\%$ \\
\hline $20-30$ & 46 & 37,4 & Masculino & 45 & 36,6 \\
\hline $31-40$ & 43 & 35 & Femenino & 78 & 63,4 \\
\hline $41-50$ & 19 & 15,4 & Total & 123 & 100 \\
\hline $51-60$ & 12 & 9,8 & & & \\
\hline mayor de 60 & 3 & 2,4 & & & \\
\hline Total & 123 & 100 & & & \\
\hline Profesión & $\mathbf{n}$ & $\%$ & Nacionalidad & $\mathbf{n}$ & $\%$ \\
\hline Auxiliar de Aseo & 5 & 4,1 & Chileno(a) & 113 & 91,9 \\
\hline Administrativo & 16 & 13 & Colombiano (a) & 1 & 0,8 \\
\hline Enfermería & 25 & 20,3 & Costarricence & 1 & 0,8 \\
\hline Kinesiología & 2 & 1,6 & Ecuatoriano(a) & 3 & 2,4 \\
\hline Medicina & 16 & 13 & Mexicano (a) & 1 & 0,8 \\
\hline Odontología & 14 & 11,4 & Peruano(a) & 1 & 0,8 \\
\hline $\begin{array}{l}\text { Técnico en Enfemería / } \\
\text { Técnico en Odontología }\end{array}$ & 36 & 29,3 & Uruguayo(a) & 1 & 0,8 \\
\hline No identificado & 9 & 7,3 & Venezolano(a) & 2 & 1,6 \\
\hline Total & 123 & 100 & Total & 123 & 100,0 \\
\hline
\end{tabular}

Tabla 2: Patologías base y Hábitos entre Funcionarios Unidad de Emergencia Hospital Barros Luco Trudeau. 2020. $n=123$

\begin{tabular}{|ccc|}
\hline Enfermedad & $\mathbf{n}$ & $\%$ \\
\hline Diabetes & 5 & 4,1 \\
Hipertensión Arterial & 9 & 7,3 \\
Asma & 4 & 3,3 \\
Obesidad & 12 & 9,8 \\
Sin Identificar & 102 & 82,9 \\
Fumadores & 53 & 43,1 \\
\hline
\end{tabular}

Tabla 3: Contacto con individuos COVID-19 positivos Unidad de Emergencia Hospital Barros Luco Trudeau. 2020. $n=123$

\begin{tabular}{|ccc|}
\hline $\begin{array}{l}\text { ¿Ha tenido contacto con individuos positivos } \\
\text { COVID-19? }\end{array}$ & n & $\%$ \\
\hline Sí & 112 & 91,1 \\
No & 7 & 5,7 \\
No se & 4 & 3,3 \\
Total & 123 & 100,0 \\
\hline
\end{tabular}

Tabla 4: Síntomas y Signos presentados sospechosos de COVID-19 y porcentaje de notificación Unidad de Emergencia, Hospital Barros Luco Trudeau. 2020. $n=123$

\begin{tabular}{|c|c|c|c|c|c|}
\hline $\begin{array}{l}\text { ¿Qué signo/síntomas ha presentado según } \\
\text { lo establecido por OMS? }\end{array}$ & $\mathrm{n}$ & $\%$ & $\begin{array}{l}\text { ¿Ha sido notificado alguna vez como } \\
\text { caso COVID-19 positivo? }\end{array}$ & $\mathbf{n}$ & $\%$ \\
\hline Fiebre & 27 & 22,0 & Sí & 48 & 39,0 \\
\hline Tos Seca & 27 & 22,0 & No & 75 & 61,0 \\
\hline Cansancio & 42 & 34,1 & Total & 123 & 100,0 \\
\hline Mialgia & 39 & 31,7 & & & \\
\hline Odinofagia & 26 & 21,1 & & & \\
\hline Diarrea & 17 & 13,8 & & & \\
\hline Conjuntivitis & 5 & 4,1 & & & \\
\hline Cefalea & 51 & 41,5 & & & \\
\hline Anosmia - Disgeusia & 24 & 19,5 & & & \\
\hline Cianosis & 4 & 3,3 & & & \\
\hline Dolor torácico & 19 & 15,4 & & & \\
\hline Dificultad respiratoria & 15 & 12,2 & & & \\
\hline Dificultad motora & 5 & 4,1 & & & \\
\hline Dolor de oído & 1 & 0,8 & & & \\
\hline Sin síntomas & 57 & 46,3 & & & \\
\hline
\end{tabular}


Moris et al.

Tabla 5: Uso de test por Inmunoglobulinas y PCR

Unidad de Emergencia, Hospital Barros Luco Trudeau. 2020. $n=123$

\begin{tabular}{|c|c|c|c|c|}
\hline \multicolumn{5}{|c|}{ ¿Se ha realizado test rapido o PCR? } \\
\hline \multirow{2}{*}{ Número de veces } & \multicolumn{2}{|c|}{ Test Rápido } & \multicolumn{2}{|c|}{ PCR } \\
\hline & $\mathrm{n}$ & $\%$ & $\mathrm{n}$ & $\%$ \\
\hline 1 & 54 & 43,9 & 46 & 37,4 \\
\hline 2 & 36 & 29,3 & 23 & 18,7 \\
\hline 3 o más & 10 & 8,1 & 22 & 17,9 \\
\hline No se ha realizado & 23 & 18,7 & 32 & 26,0 \\
\hline Total & 123 & 100,0 & 123 & 100,0 \\
\hline
\end{tabular}

Tabla 6: EPP utilizadas, origen y secuencia de instalación y retiro Unidad de Emergencia Hospital Barros Luco Trudeau. 2020. $n=123$

\begin{tabular}{|c|c|c|c|c|c|c|c|c|}
\hline $\begin{array}{l}\text { ¿Qué EPP usa en su lugar } \\
\text { de trabajo? }\end{array}$ & $\mathrm{n}$ & $\%$ & $\begin{array}{l}\text { ¿Usa secuencia de } \\
\text { instalación de EPP? }\end{array}$ & $\mathbf{n}$ & $\%$ & $\begin{array}{l}\text { ¿Usa secuencia de retiro } \\
\text { de EPP? }\end{array}$ & $\mathbf{n}$ & $\%$ \\
\hline Mascarillas Quirúrgicas & 118 & 95,9 & Siempre & 74 & 60,2 & Siempre & 84 & 68,3 \\
\hline Mascarilla N95 & 50 & 40,7 & Casi siempre & 47 & 38,2 & Casi siempre & 38 & 30,9 \\
\hline Mascarilla KN95 & 109 & 88,6 & Casi nunca & 2 & 1,6 & Casi nunca & 1 & 0,8 \\
\hline Mascarilla no desechable & 1 & 0,8 & Nunca & 0 & 0,0 & Nunca & 0 & 0,0 \\
\hline Antiparras & 62 & 50,4 & & & & & & \\
\hline Escudo facial & 92 & 74,8 & & & & & & \\
\hline $\begin{array}{l}\text { Mascara facial con } \\
\text { respirador (full face) }\end{array}$ & 4 & 3,3 & & & & & & \\
\hline Gorro desechable & 28 & 22,8 & & & & & & \\
\hline Gorro no desechable & 80 & 65,0 & & & & & & \\
\hline Guantes & 111 & 90,2 & & & & & & \\
\hline Pechera & 103 & 83,7 & & & & & & \\
\hline Overol & 4 & 3,3 & \multicolumn{4}{|c|}{$\begin{array}{l}\text { ¿Los EPPs usados fueron otorgados por el } \\
\text { centro de salud en un } 100 \% ?\end{array}$} & $\mathrm{n}$ & $\%$ \\
\hline Cubrecalzados & 6 & 4,9 & \multicolumn{4}{|c|}{ Sí } & 72 & 58,5 \\
\hline Ninguno & 0 & 0,0 & \multicolumn{4}{|c|}{ No } & 51 & 41,5 \\
\hline
\end{tabular}

\section{Discusión}

La enfermedad por coronavirus (COVID-19) es una enfermedad infecciosa emergente, de proporciones pandémicas, constituyendo un problema de salud global en la actualidad, dado la rapidez de progresión de la enfermedad (Ong et al., 2020). El primer informe emitido por China el 31 de diciembre del 2019, el cual daba a conocer la aparición de una enfermedad de virus desconocido. El 7 de enero del 2020, China informa a la OMS de que la patología notificada anteriormente era producida por un nuevo brote de coronavirus. El 11 de febrero de 2020 la OMS denomina la enfermedad como COVID-19 (Rothan et al., 2020; Wong et al., 2020).
Transcurridos 404 días desde la confirmación del primer caso en Chile (3 de marzo, 2020), el país reporta 1076499 casos y 24346 personas fallecidas a causa de COVID-19. 3.155 pacientes se encuentran hospitalizados en la actualidad (Valdivia, 2020).

El Minsal define contacto estrecho como toda persona que ha estado expuesta a un caso confirmado o probable de COVID-19, desde los 2 días antes y hasta 11 días después del inicio de síntomas (Minsal, 2021).

De las 123 encuestas realizadas en la SU, en relación con el uso de EPP en los funcionarios, la mayoría de ellos tiene una secuencia predeterminada para su instalación y retiro. Los más utilizados son mascarillas quirúrgicas, mascarilla KN 95 y guantes. 
En los inicios de la pandemia, la infección de los trabajadores de la salud llegaba al 29,0\%, y posteriormente disminuyó drásticamente. Esta disminución en la tasa de infección probablemente sea una consecuencia del uso de las medidas y uso de EPP implementados para proteger adecuadamente a los trabajadores de la salud (Stewart et al., 2020). Es importante mencionar que el uso de los EPP es a menudo decidido individualmente por los trabajadores de salud, independiente de los protocolos establecidos por los centros hospitalarios.

Un estudio realizado en Singapur a trabajadores del área de la salud de primera línea en los hospitales principales, tenían el mandato de usar los EPP mientras atendían a los pacientes con COVID-19 sospechosos o confirmados, lo que implica la utilización de mascarillas N95, gafas protectoras, batas, guantes quirúrgicos y el uso de mascara facial con respirador. En la práctica diaria, los funcionarios sanitarios a menudo sienten que ponerse el EPP es engorroso e incómodo (Wong et al., 2020), especialmente si es necesario un período prolongado de exposición a dicho equipo durante los brotes de enfermedades infecciosas emergente (Jin et al., 2020; Wong et al., 2020).

Por otra parte, la presencia de enfermedades crónicas entre el personal de salud es algo que preocupa al momento de atender a pacientes portadores de una infección viral con consecuencias graves como es la infección por SARS- CoV-2. Dentro de las patologías de base estudiadas en los funcionarios, se destaca que el grupo sin enfermedades crónica fue el grupo predominante (mayor al 74,0\%), comparado con el grupo portador de alguna patología crónica. Dentro de las patologías prevalentes están la obesidad con un 9,4\%. Además, el 43,0 \% de los individuos, indicaron que eran fumadores, lo que se asocia a un peor pronóstico de evolución de la enfermedad por COVID-19.

La obesidad se asocia a una peor respuesta inmune y un mal pronóstico para las infecciones respiratorias, como se puso en evidencia durante la epidemia de influenza tipo $A(\mathrm{H} 1 \mathrm{~N} 1)$ en el 2009. Basalmente las personas con obesidad se asocian a un bajo grado de inflamación crónica; tienen concentraciones disminuidas de adiponectinas antiinflamatorias y elevadas de citocinas proinflamatorias como la interleucina 6, el factor de necrosis tumoral alfa o la leptina, producidos en el tejido graso visceral y subcutáneo abdominal, especialmente en varones, todos ellos asociados a mayor severidad de enfermedad por COVID 19 (Luzi \& Radaelli, 2020).

Se ha implicado una asociación entre el tabaquismo y un peor pronóstico de la enfermedad en pacientes con COVID-19, pero necesitan más investigaciones para validar estos hallazgos iniciales y también establecer los mecanismos subyacentes a la presentación de síntomas más graves de COVID-19 en fumadores (Shastri et al., 2021).
Por otro lado, la diabetes ha demostrado de forma consistente ser un factor de riesgo de mal pronóstico en pacientes con COVID-19. La probabilidad de desarrollar un cuadro severo e ingresar en las unidades de cuidados intensivos ( $\mathrm{UCl}$ ) es de más del doble en las personas con diabetes (Bellido \& Pérez, 2020; Roncon et al., 2020). La mortalidad descrita es hasta 3 veces superior $(21,0-31,0 \%)$ (Bellido \& Pérez, 2020; Shing et al., 2020).

La existencia de base en la diabetes es un estado de inflamación crónica, el deterioro de la respuesta inmunológica y la alteración de la coagulación podrían estar entre los mecanismos fisiopatológicos subyacentes que contribuyen al aumento de la morbimortalidad de la COVID-19 en las personas con diabetes (Bellido \& Pérez, 2020). Se ha descrito, además, un posible daño directo al páncreas por el SARS-CoV-2, que podría empeorar la hiperglicemia, o incluso inducir la aparición de diabetes transitoria (Bellido \& Pérez, 2020).

Se observó que 39,0\% de los funcionarios fue notificado como COVID-19 positivo. Donde el 46,3\% no presentó ningún síntoma, no obstante, al considerar sintomatología prevalente según lo establecido por la OMS fueron dolor de cabeza $(41,5 \%)$, seguido de cansancio $(34,1 \%)$ y odinofagia $31,7 \%$.

El personal de salud que atiende a los pacientes con COVID-19 constituye un grupo de alto riesgo de contagio, de los casos confirmados en China 3,8\% corresponden a funcionarios de la salud, 10,0 - 20,0\% de los casos reportados en diferentes áreas geográficas de Italia, 6,0\% de los casos reportados en Holanda, 20,0\% de los casos reportados en España y 10,0 - 20,0\% de los casos reportados en diferentes localidades de Estados Unidos (Umanzor et al., 2020).

Por otro lado, un $24,1 \%$ de los casos declarados a la Red Nacional de Vigilancia Epidemiológica de España desde el inicio de la alerta por SARS-CoV-2 hasta el 10 de marzo del año 2020 fueron profesionales de la salud. Siendo la sintomatología más frecuente de tipo inespecífico (mal estado general, artralgias y/o astenia), seguida de tos, febrícula, cefalea, odinofagia y fiebre de más de $38^{\circ} \mathrm{C}$, correspondiendo la combinación de síntomas más frecuente, compromiso del estado general y tos (Bonet et al., 2021).

Este espectro de sintomatología inicial inespecífica se asemeja al descrito en una serie de 48 trabajadores sanitarios en E.E.U.U, en el cual, los síntomas más frecuentes fueron tos (50,0\%), fiebre $(41,7 \%)$ y las mialgias (35,4\%) (Chow et al., 2020).

El riesgo de infección por coronavirus SARS-CoV-2 en el personal de salud depende de la incidencia de la enfermedad en la población general, las medidas de contención implementadas, la disponibilidad de equipos de protección personal y la calidad de los programas de vigilancia epidemiológica implementados 
en los diferentes países (Umanzor et al., 2020). De este modo, el riesgo de contagio del personal de salud ha fluctuado entre $4,0 \%$ y 20,0\% de los casos reportados en diferentes áreas geográficas a nivel mundial (Kluytmans et al., 2020).

Las intervenciones para prevenir la transmisión del COVID-19 incluyen: la ampliación de los criterios de detección basados en síntomas, suspensión de actividades del personal de salud sintomático, facilitación de pruebas en personal de salud sintomático, y la creación de políticas de licencia por enfermedad que no sean punitivas, flexibles y coherentes con las pautas de salud pública. El uso de mascarillas faciales por parte de todos los funcionarios para el control de la fuente podría prevenir la transmisión de personal sanitario levemente sintomáticos y asintomáticos. Esto puede ser particularmente importante en entornos de centros de atención a largo plazo y regiones con transmisión comunitaria generalizada (Chow et al., 2020).

\section{Conclusiones}

En un momento en el que la capacidad de respuesta del sistema sanitario depende, entre otros factores, de la salud de sus trabajadores, es de gran importancia reconocer de forma precoz cuál es el patrón sintomático al diagnóstico que se asocia con un resultado PCR (+). Se puede deducir de los resultados obtenidos, que los funcionarios de salud disponían del equipo de EPP necesario para evitar contagios por COVID-19, sin embargo, la utilización de este fue inadecuada, lo que se traduce en un número significativo de contagios. Este estudio contribuye a la importancia en la utilización de EPP por parte de funcionarios de la salud, para aumentar los cuidados durante la pandemia de COVID-19, además de describir un patrón de sintomatología más frecuente en los profesionales sanitarios. Síntomas como: compromiso del estado general, mialgia, tos seca, fiebre y/o cefalea deberían ser considerados para el diagnóstico precoz.

En un contexto en el que, en el servicio, ya se estaban aplicando medidas de contención, este estudio ha permitido constatar la presencia de posibles falencias dentro de la utilización de elementos de protección personal dentro de los funcionarios de la salud de la Unidad de Emergencia.

\section{Referencias}

Bellido V. \& Pérez A. (2020). Consecuencias de la COVID-19 sobre las personas con diabetes. Endocrinología, Diabetes y Nutrición, 67, 355-356.

Bogoch I, Watts A, Thomas-Bachli A, Huber C, Kraemer M. \& Khan K. (2020). Pneumonia of unknown aetiology in Wuhan, China: potential for international spread via commercial air travel. Journal of Travel Medicine 27, 1-3.
Bonet M, Villarte, L. \& Barbé E. (2020). Initial symptomatology of SARS-CoV-2 infection in healthcare personnel: what have we learned. Atención Primaria 53, 8-9.

Carlos W, De la Cruz C, Cao B, Pasnick S. \& Jamil S. (2020). Novel Wuhan (2019-nCoV) coronavirus. American Journal of Respiratory and Critical Care Medicine 201, 7-8.

Chow E, Schwartz N, Tobolowsky F, Zacks R, Huntington-Frazier M, Reddy S. \& Rao K. (2020). Symptom Screening at Illness Onset of Health Care Personnel with SARS-CoV-2 Infection in King County, Washington. Journal of the American Medical Association 323, 2087-2089.

Cook T. (2020). Personal protective equipment during the coronavirus disease (COVID) 2019 pandemic - a narrative review. Anaesthesia 75, 920-927.

European Centre for Disease Prevention and Control (2020). Increased transmission of COVID-19 in the EU/EEA and the UK. ECDC. Accedido en: http://www.astrid-online.it/static/upload/a519/ a519ff9ba00127c29a183a6725d59f0f.pdf el 20 de marzo del 2021.

Hellewell J, Abbott S, Gimma A, Bosse N, Jarvis C. \& Russell T. (2020). Feasibility of controlling COVID-19 outbreaks by isolation of cases and contacts. Lancet Glob Heal 8, 488-496.

Huang C, Wang Y, Li X, Ren L, Zhao J. \& Hu Y. (2020). Características clínicas de pacientes infectados con el nuevo coronavirus 2019 en Wuhan, China. Lancet 395, 497-506.

Equipo COVID-19, RENAVE, CNE. (2020). Informe sobre la situación de COVID-19 en personal sanitario en España a 21 de mayo de 2020. Instituto de Salud Carlos III. Accedido en: https://www.isciii.es/ QueHacemos/Servicios/VigilanciaSaludPublicaRENAVE/EnfermedadesTransmisibles/Documents/INFORMES/Informes\%20COVID-19/ COVID-19\%20en\%20Espa\%C3\%B1a.\%20Situaci\%C3\%B3n\%20 en\%20Sanitarios\%20a\%2021\%20de\%20mayo\%20de\%202020. pdf el 20 de marzo del 2021.

Jin Y, Cai L, Cheng Z, et al. (2020). A rapid advice guideline for the diagnosis and treatment of 2019 novel coronavirus (2019-nCoV) infected pneumonia. Military Medical Research 7, 4.

Kluytmans M, Buiting A, Pas S, Bentvelsen R, van den Bijllaardt W, van Oudheusden A, et al. (2020). SARS-CoV-2 infection in 86 healthcare workers in two Dutch hospitals in March. Journal of the American Medical Association Netw Open 3, e209673.

Li Q, Guan X, Wu P, Wang X, Zhou L. \&Tong Y. (2020). Dinámica de transmisión temprana en Wuhan, China, de una nueva neumonía infectada por coronavirus. The New England Journal of Medicine 382, 1199-1207. 
Moris et al.

Lu H, Stratton CW. \& Tang YW. (2020). Brote de neumonía de etiología desconocida en Wuhan China: el misterio y el milagro. Journal of Medical Virology 92, 401-402.

Luzi L. \& Radaelli MG. (2020). Influenza and obesity: Its odd relationship and the lessons for COVID-19 pandemic. Acta Diabetology 57, 759-764.

Minsal. (2021). Actualización de la definición de caso sospechoso, probable y confirmado para vigilancia epidemiologíca ante pandemia de COVID-19 en chile. Subsecretaría de Salud Pública División Planificación Sanitaria. Ministerio de Salud. Gobierno de Chile. Accedido en: https://www.minsal.cl/wp-content/uploads/2021/02/ ORD-536-04-02-2021.pdf el 04 de agosto del 2021.

Ong J, Bharatendu C, Goh Y, Tang J, Sooi K, Tan Y, Tan B, Teoh H, Ong S, Allen D. \& Sharma V. (2020). Headaches Associated With Personal Protective Equipment - A Cross-Sectional Study Among Frontline Healthcare Workers During COVID-19. Headache 60, 864-877.

Ren L, Wang Y, Wu Z, Xiang Z, Guo L. \& Xu T. (2020). Identification of a novel coronavirus causing severe pneumonia in human: a descriptive study. Chinese Medical Journal 133, 1015-1024.

Roncon L, Zuin M, Rigatelli G. \& Zuliani G. (2020). Diabetic patients with COVID-19 infection are at higher risk of ICU admission and poor short-term outcome. Journal of Clinical Virology 127, 1386-6532.

Rothan H. \& Byrareddy S. (2020). The epidemiology and pathogenesis of coronavirus disease (COVID-19) outbreak. Journal of Autoimmunity 109, 0896-8411.

Shastri MD, Shukla SD, Chong WC, Kc R, Dua K, Patel RP, Peterson GM. \& O'Toole RF. (2021). Smoking and COVID-19: What we know so far. Respiratory Medicine 176.
Singh AK, Gupta R, Ghosh A. \& Misra A. (2020). Diabetes in COVID-19: Prevalence, pathophysiology, prognosis and practical considerations. Diabetes and Metabolic Syndrome 14, 303-310.

Stewart C, Thornblade L, Diamond D, Fong Y. \& Melstrom L. (2020). Personal Protective Equipment and COVID-19: A Review for Surgeons. Annals of Surgery 272, 132-138.

Umanzor, R, Peñafiel, F., Ugarte N, Valverde A, Bravo A, Pernas S. \& Borie R. (2020). A surveillance system to detect COVID-19 infections in health care workers. Experience in 209 cases. Revista Médica de Chile 148, 724-733.

Valdivia C. (2020). Un invitado inesperado en nuestras vidas: COVID-19 en Chile. Revista Chilena de Enfermedades Respiratorias 36, 80-84.

Verbeek JH, Rajamaki B, ljaz S, Sauni R, Toomey E, Blackwood B, Tikka C, Ruotsalainen JH. \& Kilinc Balci FS. (2020). Personal protective equipment for preventing highly infectious diseases due to exposure to contaminated body fluids in health care staff. Cochrane Database of Systematic Reviews, 15, 4.

Wang W, Tang J. \& Wei F. (2020). Conocimiento actualizado del brote del nuevo coronavirus 2019 (2019-nCoV) en Wuhan, China. Journal of Medical Virology 92, 441-447.

Wong J, Leo Y. \& Tan C. (2020). COVID-19 en Singapur: experiencia actual: problemas globales críticos que requieren atención y acción. Journal of the American Medical Association 323, 1243-1244.

Zhao S, Lin Q, Ran J, Musa SS, Yang G. \& Wang W. (2020). Estimación preliminar del número básico de reproducción del nuevo coronavirus en China, de 2019 a 2020: un dato-análisis impulsado en la fase temprana del brote. International Journal of Infectious Diseases 92, 214-217.

StataCorp. 2019. Stata Statistical Software: Release 16. College Station, TX: StataCorp LLC. 